\title{
Isothermal Amplification and Multimerization of DNA by Bst DNA Polymerase
}

BioTechniques 30:852-867 (April 2001)

\author{
G.J. Hafner, I.C. Yang, L.C. \\ Wolter, M.R. Stafford, and \\ P.M. Giffard \\ Queensland University of Tech- \\ nology, Brisbane, Australia
}

\section{INTRODUCTION}

It is now established that small circular DNA molecules may serve as tem plates for isothermal in vitro rolling-circle replication reactions catalyzed by strand-displacing DNA polymerases. These reactions have been shown to yield single-stranded linear concatemeric products $(1,4,5,11)$. More recently, there have been reports that, if these reactions are carried out in the presence of two primers, one identical and one com plimentary to the circular template, then exponential amplification that yields double-stranded variable-length concatemers takes place $(12,19)$. These cascade rolling-circle amplification (CRCA) reactions appear to be limited only by the available dNTPs. From what is currently known about CRCA reactions, it is plausible that the ability of displaced strands to act both as primers and as replication substrates results in the reaction being fully self-sustaining.

Nucleic acid amplification reactions are central to DNA-based diagnostic methods, and there have been several demonstrations of rolling-circle replication-based diagnostic strategies (5, $12,18)$. Probably the most straightforward approach for using CRCA for gene or mutation detection is to make use of padlock oligonucleotides (13). These are designed to hybridize to the target such that they are circularizable with a DNA ligase. In this way, circularization of the padlock is dependent on the presence of target DNA. We have compared the ability of the stranddisplacing Bst DNA polymerase to amplify and multimerize both circular and linear padlocks. This has led to a novel strategy for the amplification of specific nucleic acid sequences and also a method for reducing the background DNA synthesis in CRCA.

\section{MATERIALS AND METHODS}

\section{Bacterial Strains}

E. coli strain PNG801 is a mutagenized derivative of the wild-type $E$. coli strain W1485 (supplied by N. Kleckner, Harvard University). The Tn 10 -derived mini-transposon 103, which encodes a kanamycin resistance determinant, was inserted into the genome by the method of Kleckner et al. (8). PNG801 is a random isolate from the mutant poolate, and the location of the transposable element in the genome is unknown. $E$. coli strain DH5 $\alpha^{\mathrm{TM}}$ was supplied by Life Technologies (Melbourne, Victoria, Australia). Genomic DNA was extracted from these strains by the SDS, EDTA, proteinase $\mathrm{K}$ method described by Silhavy et al. (17).

\section{Oligonucleotides}

All oligonucleotides were purchased from GeneWorks (Adelaide, South Australia, Australia) or Life Technologies and were synthesized using standard phosphoamidite chemistry. Oligonucleotides used as padlocks and/or synthetic amplification targets were gel purified to homogeneity by the suppliers, while all others were supplied as desalted preparations. Padlock and spacer oligonucleotides used in ligation 
reactions were either phosphorylated during synthesis or after synthesis using T4 polynucleotide kinase (15).

\section{Sensitivity Determination of CRCA Using Gel-Purified Circularized Padlock}

It should be noted that oligonucleotides that are designed to anneal to a target such that they may be circularized with a ligase we have termed "padlocks" or "padlock oligonucleotides". The term padlock probe has been avoided because we have not used the molecules as probes. The padlock B2B (150 pmol) (5'-ACGATCACGCGCTCACAGTAGAGCTGTGGCGTATCAAAG AACGAATTCTCCAGTACTACGACCTATCAATGAGTCGAGCGTGAATGACGA-3') was mixed with 150 pmol target oligonucleotide (5'-CGGGATCTGTGAGCGCGTGATCGTTCGTCA TTCACGCTCGACAGCTTG-3') in a $100-\mu \mathrm{L}$ reaction containing T4 DNA ligase buffer (Roche Diagnostics Australia, Brisbane, Queensland, Australia). The reaction was heated for 5 min at $95^{\circ} \mathrm{C}$ and then cooled slowly to room temperature. T4 DNA ligase (5 U; Roche Diagnostics Australia) was then added, and the mixture was incubated at room temperature overnight. The reaction products were electrophoresed through an $18 \%$ denaturing polyacrylamide gel, stained with ethidium bromide, and the band representing the circular molecules was excised from the gel. The molecules were eluted from the gel by the "crush and soak" method of Sambrook et al. (15).

The identity of the isolated molecules was confirmed by digestion with the restriction enzyme TaqI and differential electrophoresis mobility in denaturing $6 \%$ and $10 \%$ polyacrylamide gels. The basis for the latter technique is that circular DNA has different apparent molecular weights when electrophoresed in different concentrations of acrylamide when linear molecular weight standards are used. The concentration of the final circularized product was estimated spectrophotometrically.

The purified circular DNA was am plified in a $60-\mu \mathrm{L}$ reaction that contained 16 pmol each of primers B4C (5'-TTGATAGGTCGTAGCAT-3') and P5C (5'-AGAGCTGTGGCGTATCA-
3'), $10 \mathrm{mM} \mathrm{KCl}, 10 \mathrm{mM}\left(\mathrm{NH}_{4}\right)_{2} \mathrm{SO}_{4}$, $20 \mathrm{mM}$ Tris- $\mathrm{HCl}, \mathrm{pH} 8.8,200 \mu \mathrm{M}$ each dNTP, $2 \mathrm{mM} \mathrm{MgSO}_{4}$, and $1 \%$ Triton ${ }^{\circledR}$ $\mathrm{X}-100$. The mixture was heated to $94^{\circ} \mathrm{C}$ for $30 \mathrm{~s}$ then equilibrated at $60^{\circ} \mathrm{C}$ for 5 $\min$. At this point $4 \mathrm{U}$ Bst DNA polymerase were added, and the reactions were incubated at $60^{\circ} \mathrm{C}$ for $2 \mathrm{~h}$.

\section{Monitoring of CRCA in Real Time}

This experiment was carried out using the same batch of gel-purified circularized padlock as was used for the determination of CRCA sensitivity. The amplification conditions were also the same as described, with the exception that $15 \mu \mathrm{g}$ bovine serum albumin (BSA) and $1 \mu \mathrm{L} \mathrm{SYBR}{ }^{\circledR}$ Green I (1: 1000 dilution; Molecular Probes, Eugene, OR, USA) were included in each reaction. The reaction mixtures were denatured, and Bst DNA polymerase was added, at which point they were placed into cuvettes and then loaded into a LightCycler ${ }^{\circledR}$ (Idaho Technology, Salt Lake City, UT, USA). This was programmed to incubate the samples at $60^{\circ} \mathrm{C}$ and take fluorescence readings at 1-min intervals.

\section{Amplification from Other Templates}

Both circular and linear templates were amplified as follows. The reactions contained 16 pmol each primer, $10 \mathrm{mM} \mathrm{KCl}, 10 \mathrm{mM}\left(\mathrm{NH}_{4}\right)_{2} \mathrm{SO}_{4}, 20$ $\mathrm{mM}$ Tris- $\mathrm{HCl}, \mathrm{pH} 8.8,200 \mu \mathrm{M}$ each dNTP, $2 \mathrm{mM} \mathrm{MgSO}$, and $1 \%$ Triton $\mathrm{X}-100$. The template was added in a volume of $1-2 \mu \mathrm{L}$, and the final reaction volume was $60 \mu \mathrm{L}$. The mixture was heated to $94^{\circ} \mathrm{C}$ for $30 \mathrm{~s}$ and then equilibrated at $55^{\circ} \mathrm{C}-60^{\circ} \mathrm{C}$ for $5 \mathrm{~min}$. At this point, $4 \mathrm{U}$ Bst DNA polymerase was added, and the reactions were incubated at the equilibration temperature for $1.5-3 \mathrm{~h}$.

\section{Gel Electrophoresis and Hybridization}

The amplification reaction products were electrophoresed through $2 \%$ agarose gels in TAE or TBE buffer (15) and visualized by ethidium bromide staining. When blotting was carried out, the gels were soaked in $0.5 \mathrm{M} \mathrm{NaOH}$, $1.5 \mathrm{M} \mathrm{NaCl}$ for $30 \mathrm{~min}$, then in $1 \mathrm{M}$
Tris- $\mathrm{HCl}, \mathrm{pH} 8.0,1.5 \mathrm{M} \mathrm{NaCl}$ for 30 min. The nucleic acids were then capillary transferred to Hybond ${ }^{\circledR}-\mathrm{N}+$ membrane (Amersham Pharmacia Biotech, Sydney, NSW, Australia) according to the manufacturer's instructions. Oligonucleotide probes were 3 '-labeled with digoxigenin (DIG)-ddUTP using terminal transferase (Roche Diagnostics Australia). Membranes were prehybridized in 5-10 mL Rapid-Hyb ${ }^{\mathrm{TM}}$ buffer (Amersham Pharmacia Biotech) at $42^{\circ} \mathrm{C}$ for $30 \mathrm{~min}$ and were hybridized at $42^{\circ} \mathrm{C}$ overnight. The membranes were washed and developed using antiDIG-alkaline phosphatase conjugate and CPDStar $^{\circledR}$ (Roche Diagnostics Australia) according to the manufacturer's instructions.

\section{Cloning and Sequence Analysis}

The amplification reaction products were first purified through Wizard ${ }^{\circledR}$ PCR DNA purification columns (Promega, Madison, WI, USA). The purified products were then ligated into $\mathrm{pGEM}^{\circledR}$-T (Promega) at $16^{\circ} \mathrm{C}$ for $3 \mathrm{~h}$, and the ligated products were introduced into E. coli DH5 $\alpha$ by electroporation. Inserts were sequenced using dye-labeled terminators and a model 373A sequencer (Applied Biosystems, Foster City, CA, USA).

\section{Affinity Purification and Amplification of Circularized Padlocks Using Streptavidin-Coated Superparamagnetic Beads}

The annealing and ligation reactions were carried out in a final volume of 50 $\mu \mathrm{L}$, which contained padlock FV2 (5'pAGGAATACAGGTATTTTGTCCTTGCGCGGTGAGCTATATGGGGACTATGAATTCTAATAGGACTACCTTCTAATCTGTAAGAG-3'), synthetic target FVWT (5'-TACTTCAAGGACAAAATACCTGTATTCCTCGCCTG TCCAGGGATCTGCTCTTACAGATTAGAAGTAGTCCTATT-3'), and biotinylated spacer LigW (5' p-CAGATC$\mathrm{CC}$ (biotin-dT)GGACAGGCG-3') in ligation buffer $(20 \mathrm{mM}$ Tris- $\mathrm{HCl}, \mathrm{pH}$ 8.3, $25 \mathrm{mM} \mathrm{KCl}, 10 \mathrm{mM} \mathrm{MgCl}_{2}, 0.5$ $\mathrm{mM}$ NAD, and $0.1 \%(\mathrm{v} / \mathrm{v})$ Triton $\mathrm{X}$ 100). The mixture was heated to $94^{\circ} \mathrm{C}, 5$ U Ampligase (Epicentre Technologies; supplied by Austral Scientific, Sydney, 
NSW, Australia) were then added, and incubation at $94^{\circ} \mathrm{C}$ continued for $3 \mathrm{~min}$. The reaction was then cooled to $60^{\circ} \mathrm{C}$ and incubated at that temperature for $1 \mathrm{~h}$.

The streptavidin-coated superparamagnetic beads were supplied by Dynal Biotech (Melbourne, Victoria, Australia). The supplied bead suspension $(125 \mu \mathrm{L})$ was transferred to a $1.5-\mathrm{mL}$ microcentrifuge tube and was washed four times in $125 \mu \mathrm{L} 2 \times \mathrm{BW}$ buffer (10 $\mathrm{mM}$ Tris-HCl, $\mathrm{pH} 7.5,1 \mathrm{mM}$ EDTA, $2 \mathrm{M} \mathrm{NaCl}$ ). Pelleting of the beads was carried out using a magnetic platform. After the final wash, the beads were resuspended in $250 \mu \mathrm{L} 2 \times \mathrm{BW}$ buffer. Fifty microliters of the bead suspension were then mixed with the entire $50 \mu \mathrm{L}$ products of the annealing and ligation reactions. The mixture was incubated at room temperature for $30 \mathrm{~min}$ with occasional agitation. The beads were then pelleted using a magnetic platform, the supernatant was removed, and the beads were washed twice in $1 \times \mathrm{BW}$ buffer (5 mM Tris-HCl, $\mathrm{pH} 7.5,0.5$ mM EDTA, $1 \mathrm{M} \mathrm{NaCl}$ ), then three times in $100 \mu \mathrm{L} 0.1 \mathrm{M} \mathrm{NaOH}$ to remove any hybridized molecules, then twice in TE buffer $(10 \mathrm{mM}$ Tris- $\mathrm{HCl}$, pH 8.0, 1 mM EDTA). For each wash, the beads were suspended in the relevant buffer for $5 \mathrm{~min}$ and then pelleted using a magnetic platform. After the washes, the beads were suspended in $50 \mu \mathrm{L}$ TE buffer. One microliter of the suspension was used as a template in amplification reactions, as described above, using primers PadFV3 (5'-GAAATTCATAGTCCCC-3') and PadFV4 (5'-CGCGGTGAGCTATATAT-3').

\section{Affinity Purification and Amplifica- tion of Circularized Padlock Using Streptavidin-Coated Microplates}

This procedure also made use of the padlock FV2, the biotinylated spacer
LigW, and the amplification primers PadFV 3 and PadFV4. The targets used were the synthetic oligonucleotide FVWT, human genomic DNA, and $E$. coli genomic DNA (negative control). These oligonucleotides are based around the Factor V Leiden mutation, and the human DNA used was homozygous normal at this site.

Annealing and ligation reactions containing padlock, biotinylated spacer oligonucleotide, and target DNA were carried out as described above. The entire reaction products were transferred to streptavidin-coated microplates (NEN ${ }^{\circledR}$ Life Science Products, Boston, MA, USA) and incubated at room temperature for $30 \mathrm{~min}$. The wells were then washed twice with $1 \times \mathrm{BW}$, twice with $0.15 \mathrm{M} \mathrm{NaOH}$, once with $1 \times \mathrm{BW}$, 12 times with phosphate-buffered saline containing $0.05 \% \quad(\mathrm{v} / \mathrm{v})$ Tween ${ }^{\circledR} 20$ (PBST) (15), and finally once with TE. All washes were $200 \mu \mathrm{L}$ 


\section{Research Report}

in volume. The amplification reaction components (as described above) were then added to the wells, and the microplates were then heated at $98^{\circ} \mathrm{C}$ for $3 \mathrm{~min}$, cooled to $60^{\circ} \mathrm{C}$, and $4 \mathrm{U}$ Bst DNA polymerase were added to each well. The microplates were then incubated at $60^{\circ} \mathrm{C}$ for $90 \mathrm{~min}$

\section{RESULTS}

Minimal Circular Template Is

Required for the Synthesis of

Abundant Linear Amplimer by

\section{Bst DNA Polymerase}

We are interested in the diagnostic potential of CRCA. It is now clear that if short single-stranded circles of DNA are combined with Bst DNA polymerase, two primers, and dNTPs in a suitable buffer and incubated at approximately $60^{\circ} \mathrm{C}$, a self-sustaining exponential amplification reaction takes place $(12,19)$. We have attempted to accurately determine the sensitivity of this reaction. A single-stranded circular

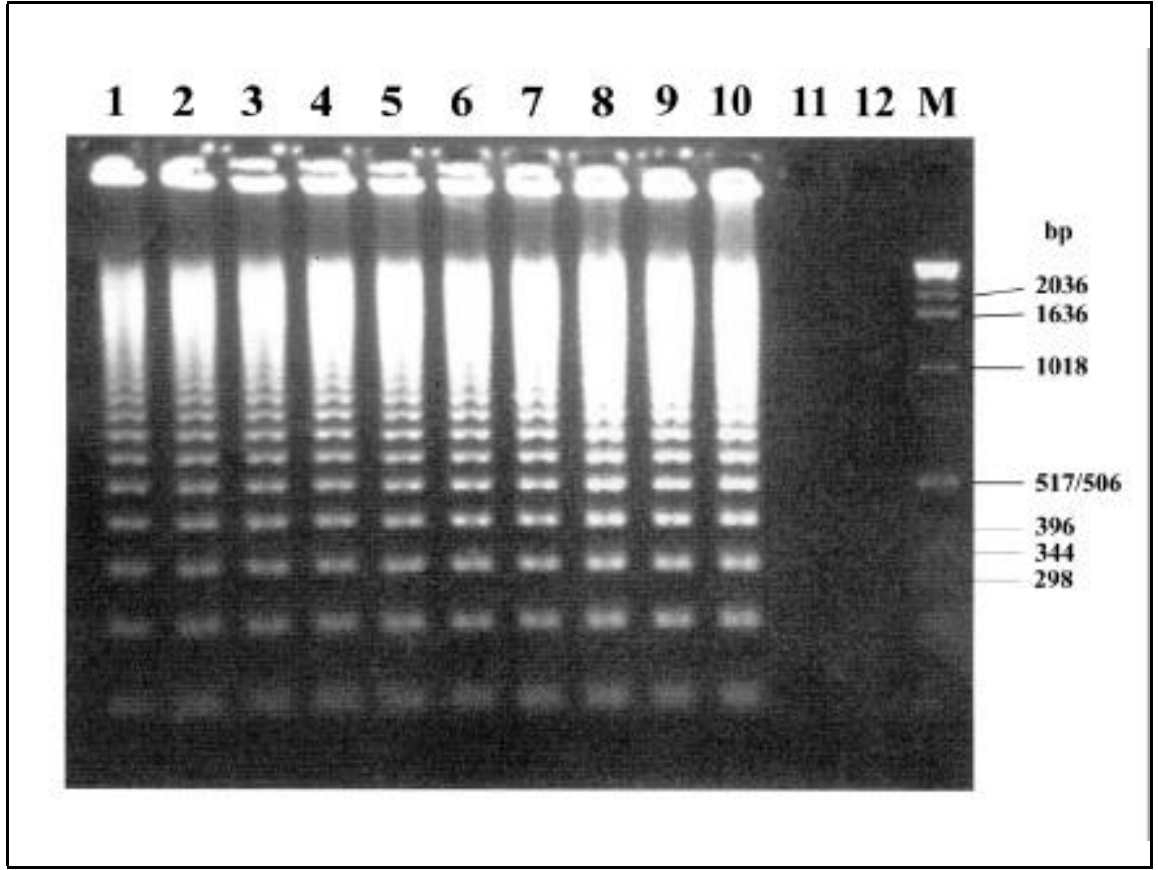

Figure 1. Sensitivity of CRCA of gel-purified circular template DNA. Dilutions of a gel-purified single-stranded circular template were subject to amplification for $2 \mathrm{~h}$, and the products were analyzed by electrophoresis. The amount of template in each reaction was extrapolated from spectrophotometric analysis of the undiluted template preparation. Lanes 1-11 show the CRCA products from a tenfold dilution series of template starting at $8 \times 10^{9}$ molecules (lane 1) and terminating at 0.8 molecules (lane 11). Lane 12, no template. Lane M, molecular weight makers.

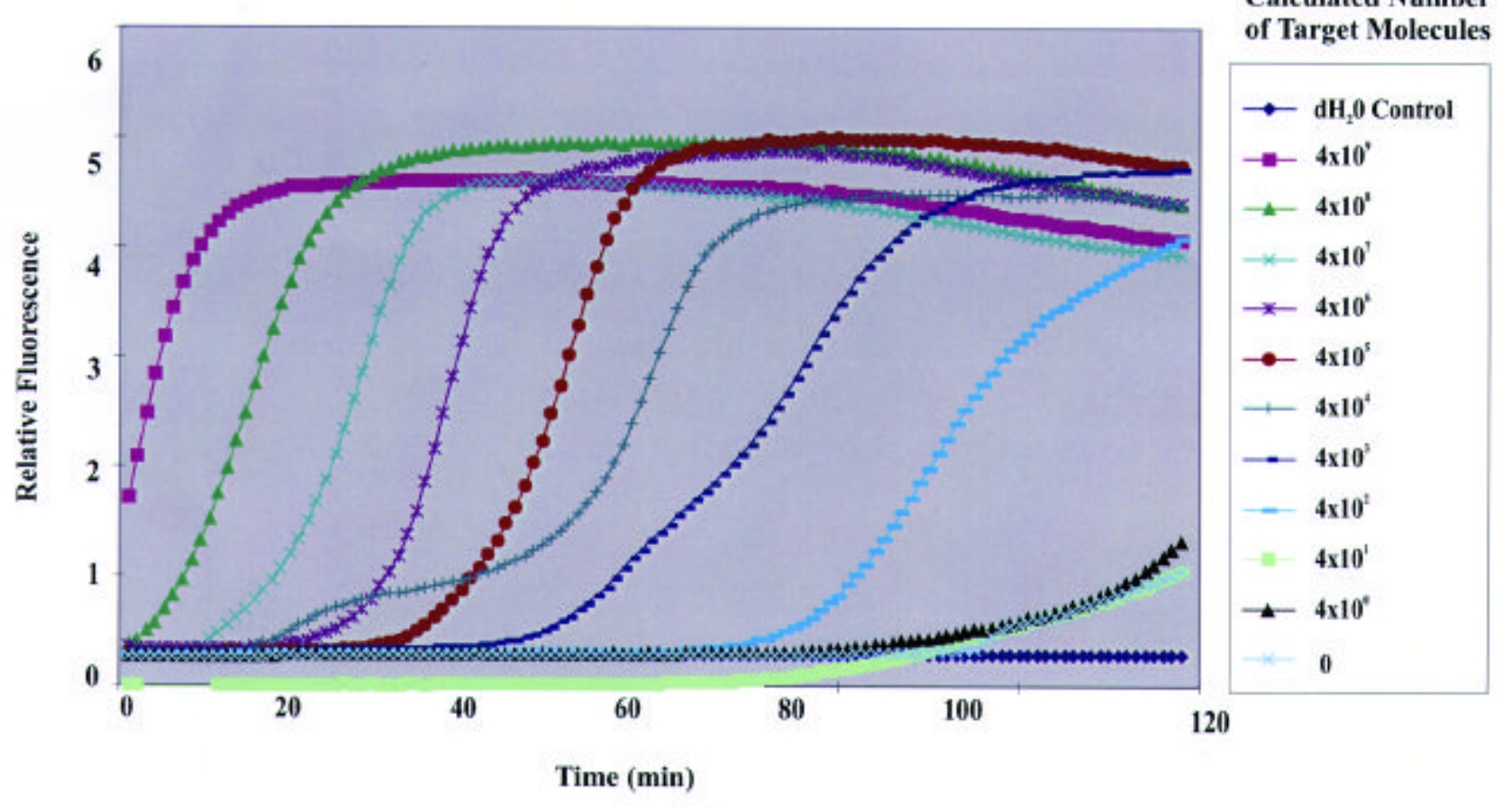

Figure 2. Real-time monitoring of CRCA. CRCA reactions using differing amounts of template were carried out in a LightCycler, and the accumulation of dsDNA was followed through measurement of SYBR Green I fluorescence. A. Raw output from the LightCycler. 


\section{Research Report}

template was constructed through hybridization of a padlock to a synthetic template and ligation of the padlock ends. The circular product was then gel-purified, and the abilities of various dilutions of this circular molecule to serve as rolling-circle templates were determined. It was found that approximately 10 circular molecules were all that was required for amplification to take place (Figure 1). As expected, the reactions yielded large quantities of variable-length concatemers. The self- sustaining nature of the reactions was evident from the lack of any direct relationship between the amounts of circular template DNA and product.

\section{Relationship between Template Concentration and Reaction Kinet- ics in CRCA Is Similar to PCR}

In the case of PCR, target quantitation is often done by determining the number of amplification cycles before a predefined quantity of amplimer is syn- thesized. To determine if CRCA is amenable to a similar quantitation strategy, CRCA reactions containing different dilutions of template were carried out in a LightCycler. This device measures the accumulation of dsDNA in real time by making use of the doublestranded nucleic acid-specific fluorescent dye SYBR Green I. Figure 2 shows that there is a clear relationship between the $\log$ of the template concentration and the time taken to reach a threshold level of amplimer.

A

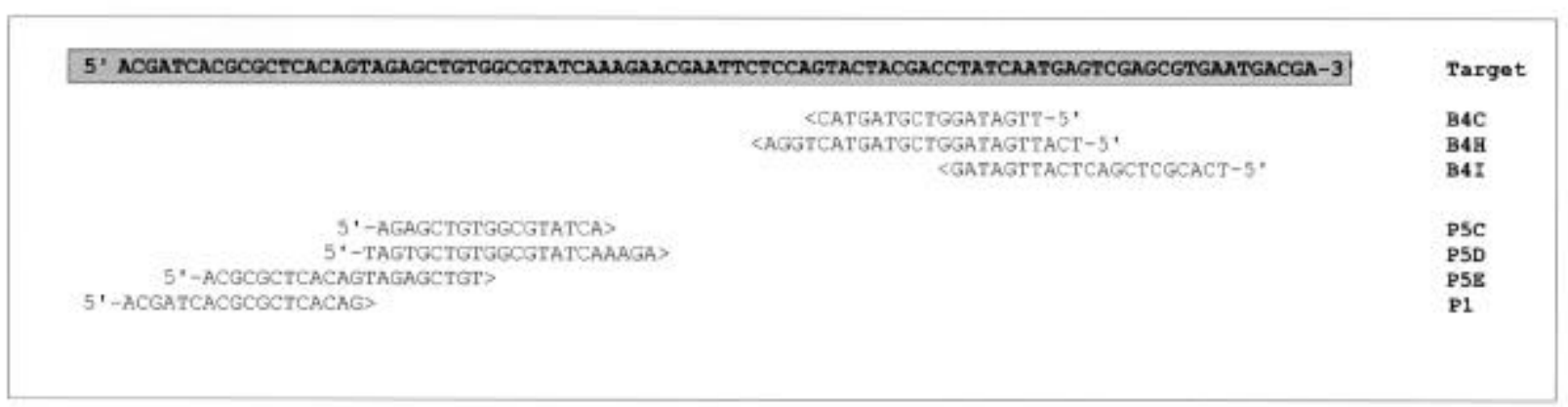

B

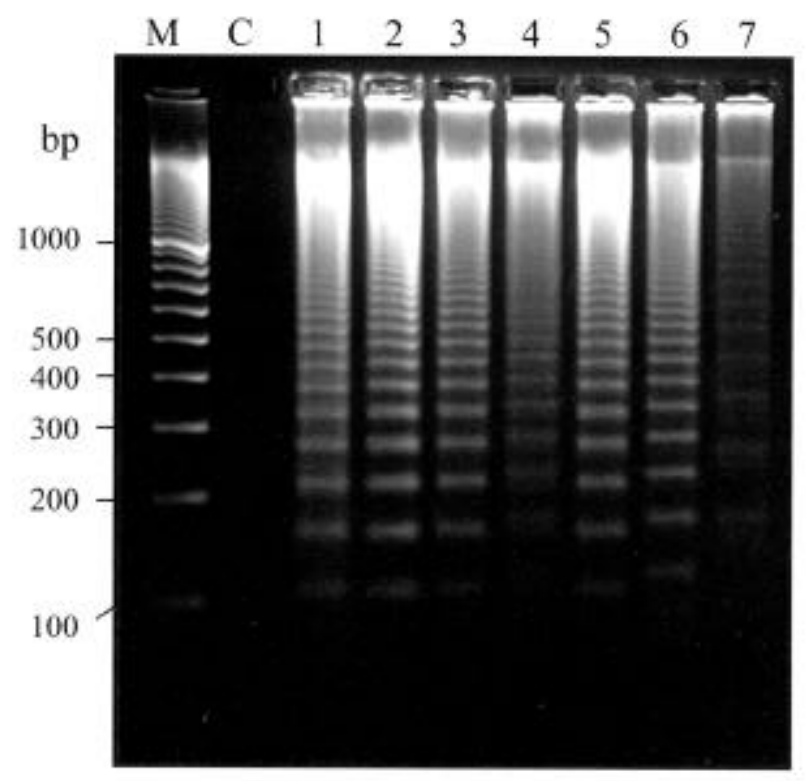

Figure 3. Products of LIMA reactions using different primer sets. LIMA reactions were carried out using $1 \mathrm{pmol}$ target and $16 \mathrm{pmol}$ each primer and $3 \mathrm{~h}$ incubation at $60^{\circ} \mathrm{C}$. (A) Sequences of the target (B2B padlock) and primers. (B) Ten microliters of the reaction products were electrophoresed in $2 \%$ agarose. Lane M, molecular weight markers; lane C, no template; lane 1, primers $\mathrm{P} 1$ and $\mathrm{B} 4 \mathrm{C}$; lane 2, primers $\mathrm{P} 5 \mathrm{C}$ and $\mathrm{B} 4 \mathrm{C}$; lane 3, primers P5C and B4H; lane 4, primers $\mathrm{P} 5 \mathrm{C}$ and $\mathrm{B} 4 \mathrm{I}$; lane 5, primers $\mathrm{P} 5 \mathrm{D}$ and $\mathrm{B} 4 \mathrm{C}$; lane 6, primers $\mathrm{P} 5 \mathrm{D}$ and $\mathrm{B} 4 \mathrm{I}$; and lane 7, primers P5E and B4I. 


\section{Research Report}

In the Presence of Linear Template DNA, CRCA Reactions Are Prone to Background

Padlocks are designed to anneal to the target sequence such that they are circularizable with a conventional DNA ligase (13). As circularization can only occur in the presence of the target, this provides a strategy for CRCA-based gene detection or, if ligation can be made sufficiently specific, a strategy for mutation diagnosis.

In practice, however, it is very difficult to devise reaction conditions in which DNA synthesis does not occur in the absence of target DNA. Electrophoresis of this background material com monly revealed the "ladder" effect of CRCA products that is indicative of variable-length multimers (data not shown).

\section{Linear Molecules May Be Amplified under CRCA Reaction Conditions}

The background problems experienced lead us to hypothesize that circular templates are not necessary for exponential DNA amplification under CRCA reaction conditions. This was shown to be the case with seven different synthetic 90-mer templates (data not shown). A series of "linear template"

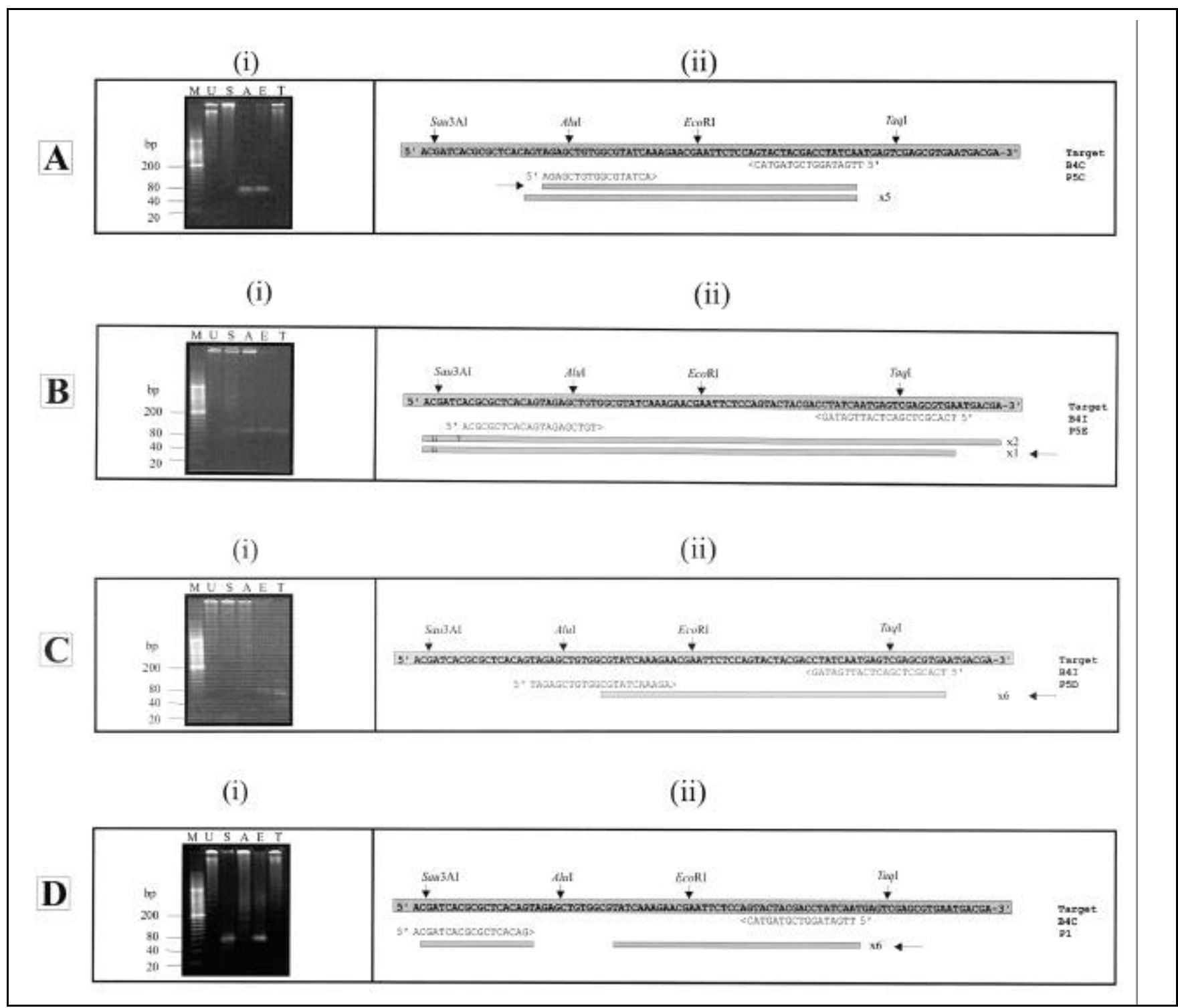

Figure 4. Analysis of LIMA products. The products of four LIMA reactions (A-D) were analyzed by restriction digestion/electrophoresis and sequence determination. The reactions were carried out using $16 \mathrm{pmol}$ each primer and 1 pmol target (padlock B2B) and were incubated at $60^{\circ} \mathrm{C}$ for $3 \mathrm{~h}$. The $2 \%$ agarose gels are shown in A(i)-D(i). Lane M, molecular weight markers; lane U, undigested reaction products; lane S, Sau3AI-digested amplimer; lane A, AluI-digested am plimer; lane E, EcoRI-digested amplimer; lane T, TaqI-digested amplimer. The reaction products were also cloned, and partial sequence was obtained from one clone from each LIMA reaction. The relationships between the repeating units in the clone sequences and the target and primer sequences are depicted in A(ii)$\mathrm{D}$ (ii). The boxes show the position of the repeating units. The numbers to the right of the boxes show the number of consecutive repeats in the sequence obtained. The arrows show the directions of the sequence reads. Mutations with respect to the target are indicated within the boxes [B(ii) only]. The discontinous box in $\mathrm{D}$ (ii) indicates a deletion in the repeating unit with respect to the target. 
reactions was carried out and the am plimers analyzed in detail (Figure 3A). Each reaction contained the same tem plate and different primer sets.

Electrophoresis of the reaction products revealed that all primer sets promoted DNA synthesis and that in all cases the banding was consistent with variable-length multimers (Figure 3B). Spectrophotometric measurement indicated that amount of DNA synthesized per reaction varied from 10 to $40 \mu \mathrm{g}$.

It was concluded that linear molecules may be amplified and multimerized under isothermal conditions using $B s t$ DNA polymerase. We have termed this reaction linear target isothermal multimerization and amplification (LIMA).

\section{LIMA Products Are Derived from the Template}

Products from four reactions, each of which used the same template but different primer combinations, were characterized by restriction digestion and sequence determination. The results of these analyses are shown in Figure 4. It is clear that the reaction products are multimers of DNA derived from the target sequence. In general, the repeating units were contiguous fragments of the target sequence bounded by the $5^{\prime}$ ends of the primers, although it is apparent that introduction of deletions and point mutations sometimes occurs. In addition, the precise relationships between the primer sequences and the end points of the repeating units varied slightly.

\section{LIMA Is Less Sensitive than CRCA}

The synthetic target $\mathrm{B} 2 \mathrm{~B}$ and primers $\mathrm{B} 4 \mathrm{C}$ and $\mathrm{P} 5 \mathrm{C}$ were used to determine the sensitivity of the LIMA reaction. It was found that the target detection limit was 0.1 fmol $\left(6.02 \times 10^{7}\right.$ molecules $)$ (Figure 5). When similar experiments were carried out using different primer/template combinations, the detection limit varied from 1 fmol to 0.01 amol (data not shown), indicating that the sensitivity is highly variable and a function of the sequences of the reactants. Despite this variability, it may be concluded that, in general, LIMA is several logs less sensitive than CRCA with respect to template concentration.

\section{LIMA Can Selectively Amplify a Target Sequence in a Bacterial Genomic Preparation}

To test the notion that LIMA may be of potential utility, its abililty to amplify a fragment of a mini-transposon inserted into the E. coli genome was determined. Primers $1 \mathrm{~F}$ and $1 \mathrm{R}$ were designed to flank a 120-bp fragment of transposable portion of the Tn 10 -derived mini-transposon 103 (8). To facilitate amplimer characterization, this fragment contains a HindIII site. Varying amounts of denatured genomic DNA from $E$. coli strains positive (pPNG801) and negative (DH5 $\alpha$ ) for this target sequence were subject to amplification for $2.5 \mathrm{~h}$. One microliter of each reaction product was treated with HindIII, and then HindIIItreated and untreated reaction products were analyzed by electrophoresis. Figure 6 shows the results.

There was no evidence for amplification when $E$. coli DH5 $\alpha$ DNA was used as the template. However, when $E$. coli PNG801 DNA was used, amplifi-

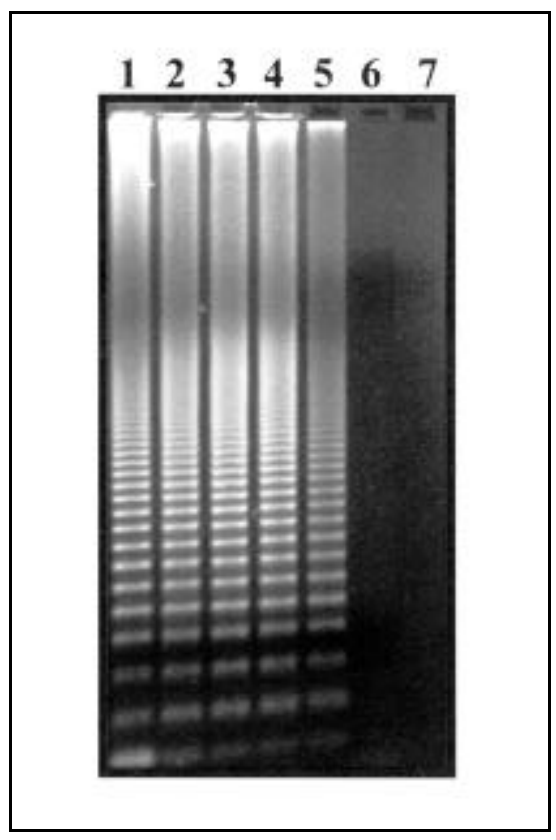

Figure 5. Sensitivity of LIMA with respect to target concentration. LIMA reactions were carried out with varying amounts of target (padlock $\mathrm{B} 2 \mathrm{~B}$ ) and primers $16 \mathrm{pmol}$ primers B4C and P5C. The reactions were incubated at $60^{\circ} \mathrm{C}$ for $3 \mathrm{~h}$. Ten microliters of amplimer were electrophoresed in $2 \%$ agarose. Lanes 1-6 show the products from reactions containing a tenfold dilution series of target starting at 1 pmol. Lane 7 represents a control reaction to which no target was added. cation took place. It was deduced from the single molecular weight species in the HindIII-treated samples that, as expected, multiple evenly spaced HindIII sites were present in the PNG801-derived amplimers. The derivation of the amplimers from the target was confirmed by hybridization with a targetderived probe (data not shown). It was concluded that specific amplification and multimerization of the target sequence had taken place.

It is noteworthy that the sizes of the repeating units varied from reaction to reaction, even though the target sequence and primers were the same. The molecular basis for this was determined by sequence analysis of three of the reaction products. Each product contained both primer binding sites and the expected 120-bp sequence between these sites. However, the repeating units from two of the reactions contained additional sequences, up to $77 \mathrm{bp}$ in size, clearly derived from the mini-transposon but from outside the primer binding sites.

\section{Removal of Linear Padlock before Amplification Reduces LIMA- Derived Background}

It is now clear that LIMA is the basis for background reactions in padlock-dependent embodiments of CRCA. Ligation of a padlock to a rare target sequence yields a mixture in which the linear padlock is present in great molar excess over the circularized padlock, thus offsetting the lower sensitivity of LIMA as compared with CRCA. In addition, LIMA will take place in the absence of target sequence. It may therefore be predicted that removal of the linear padlock before amplification would reduce the background. This was tested using a padlock and biotinylated spacer oligonucleotides designed such that the spacer hybridizes to the target between the padlock arms, thus allow ing the affinity-based separation of circularized padlock from linear padlock. This strategy was tested in two different experimental systems. In the first, a padlock/spacer set were designed to hybridize to a synthetic oligonucleotide target, and the effect of incorporating a circle purification protocol on the nature of the amplimer was determined. It may be seen in Figure 7A that the circle pu- 


\section{Research Report}

rification prevented LIMA-based am plification of the linear padlock alone (lanes 1 and 3) and eliminated LIMA derived background in the CRCA reactions (lanes 2 and 4; lane 2 contains a mixture of LIMA and CRCA products). In the second experiment, the ability of CRCA to carry out high-sensitivity gene detection was demonstrated. The protocol was designed to detect the human Factor V-encoding gene and, as expected, gave a positive result from human DNA and a negative result from $E$. coli genomic DNA (Figure 7B).

\section{DISCUSSION}

It has been shown by several researchers that strongly strand-displac- ing DNA polymerases are able to able to exponentially amplify multimeric DNA $(12,19)$. The role of rolling-circle replication in CRCA is to create a multimeric molecule that is then amplified. CRCA is therefore something of a misnomer because it may be predicted that any reaction that yields linear multimers may be substituted for the rollingcircle reaction. Although the precise mechanism of LIMA is obscure, our current model is that it is a form of in vitro evolution in which a variety of inefficient replication initiation events occurs early in the reaction. This results in Darwinian selection of molecules that are more easily replicated. As would be predicted from the rapid and exponential kinetics of CRCA, such molecules are usually multimeric. It is highly likely that the initial formation of easily amplifiable multimers is the limiting factor for the sensitivity of the reaction and, to a great extent, defines the reaction duration. The precise events that occur early in LIMA reactions will be very difficult to elucidate, but it may be deduced from the nature of the reactants and the fact that LIMA takes place at all that "illegitimate" events such as primer invasion of dsDNA must be involved. The random nature of the early stages of the reaction would account for the variabilities found with respect to both sensitivity and the end points of the multimer repeating units. The production of RNA multimers from linear templates has been previously reported by Krupp (9), and a rolling-circle-like mechanism us-

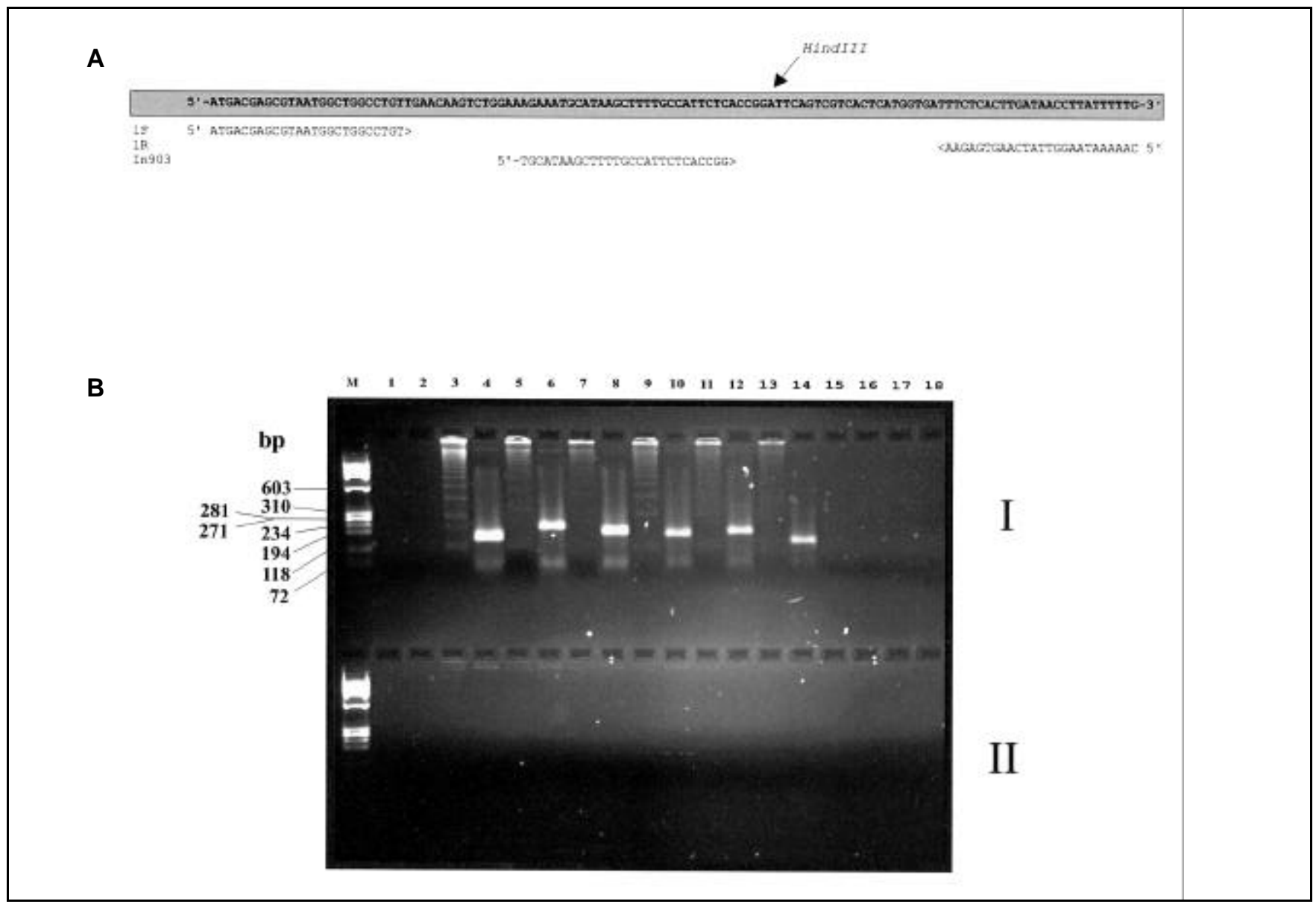

Figure 6. Use of LIMA to amplify a specific sequence from an $\boldsymbol{E}$. coli genomic preparation. (A) The sequences of the primers $1 \mathrm{~F}$ and $1 \mathrm{R}$, the target region, and the probe In903. The LIMA reactions were carried out using various quantities of E. coli genomic DNA and were incubated at $60^{\circ} \mathrm{C}$ for $2.5 \mathrm{~h}$. Untreated and $H i n d \mathrm{I}-$ II-digested reaction products were analyzed by electrophoresis in $2 \%$ agarose. (B) I, amplimers from $E$. coli PNG801 (target sequence positive); II, amplimers from E. coli DH5 $\alpha$ (target sequence negative). Lane M, molecular weight markers; lane 1, no target, untreated amplimer; lane 2, no target, HindIII-treated amplimer; lanes 3 and 5, $120 \mathrm{ng}$ target, untreated amplimer; lanes 4 and 6, $120 \mathrm{ng}$ target, HindIII-treated amplimer; lanes 7 and 9, 60 ng target, untreated amplimer; lanes 8 and 10, $60 \mathrm{ng}$ target, HindIII-treated amplimer; lanes 11 and 13, $20 \mathrm{ng}$ target, untreated amplimer; lanes 12 and 14, $20 \mathrm{ng}$ target, HindIII-treated amplimer; lanes 15 and 17, 2 ng target, untreated amplimer; lanes 16 and 18,2 ng target, HindIII-treated amplimer. The duplicates represent separate LIMA reactions. 
ing a non-covalently closed template was suggested. A similar mechanism cannot be ruled out for LIMA, although the smaller size of the repeating units in LIMA as compared with CRCA would suggest that this is not the case.

The use of LIMA in diagnostic applications is an intriguing possibility. We have demonstrated reproducible and selective amplification of a gene fragment in an E. coli genome sample, thus indicating a useful level of specificity. The variable nature of the relationship between the starting material and the reaction products, and the low sensitivity as compared with CRCA, would suggest that applications would need to be individually optimized and that using LIMA for high sensitivity gene detection (e.g., direct diagnosis of infectious agents in clinical samples) may be problematic. However, these disadvantages may well be offset by the small number of components required and the isothermal nature of the reaction. We have not yet attempted to determine whether LIMA can be made specific to the level of single nucleotide polymorphisms, but if this proved to be feasible, then applications in the area of routine genotyping may be envisaged.

It may be inferred from the ability of $B s t$ DNA polymerase to mediate LIMA and the great molar excess of padlock over target DNA in any sensitive CRCA-based gene or mutation detection reaction that the removal of linear padlock before CRCA would reduce background problems. This was tested by affinity purifying circular DNA before amplification. As expected, background DNA synthesis was greatly reduced. The use of a biotinylated spacer would be expected to increase the effective specificity of the ligation reaction and eliminate LIMA-derived background. This is because only in the presence of the target would the spacer be brought into close proximity with the padlock and, as a result, any circular molecules formed by annealing of the padlock to a spurious target will not include the biotin molecule and will not be captured before amplification. These results are consistent with those reported by Zhang et al. (19), who have used a target purification protocol to reduce the background of DNA amplification reactions including those based on rolling-circle replication. When applied to CRCA, their protocol also would be expected to reduce the amount of linear padlock present.

It is possible that LIMA and/or CRCA may be useful in applications outside DNA-based diagnostics. It has already been established that concatemerization of response elements can enhance their affinity for transcription factors (20), and this phenomenon has been exploited for affinity chromatography and functional analysis of tran- scription factors $(2,7,16)$. Techniques previously used to generate concatemers include DNA ligation (7), direct cloning of repetitive DNA $(10,14)$, or amplification of large oligonucleotides (6). LIMA and/or CRCA could easily be used in place of these reactions. Indeed, Daubendiek and Kool (3) have recently demonstrated the production by rolling circle of self-cleaving concatemers of catalytic RNA molecules..

In summary, we have demonstrated that Bst DNA polymerase readily multi-

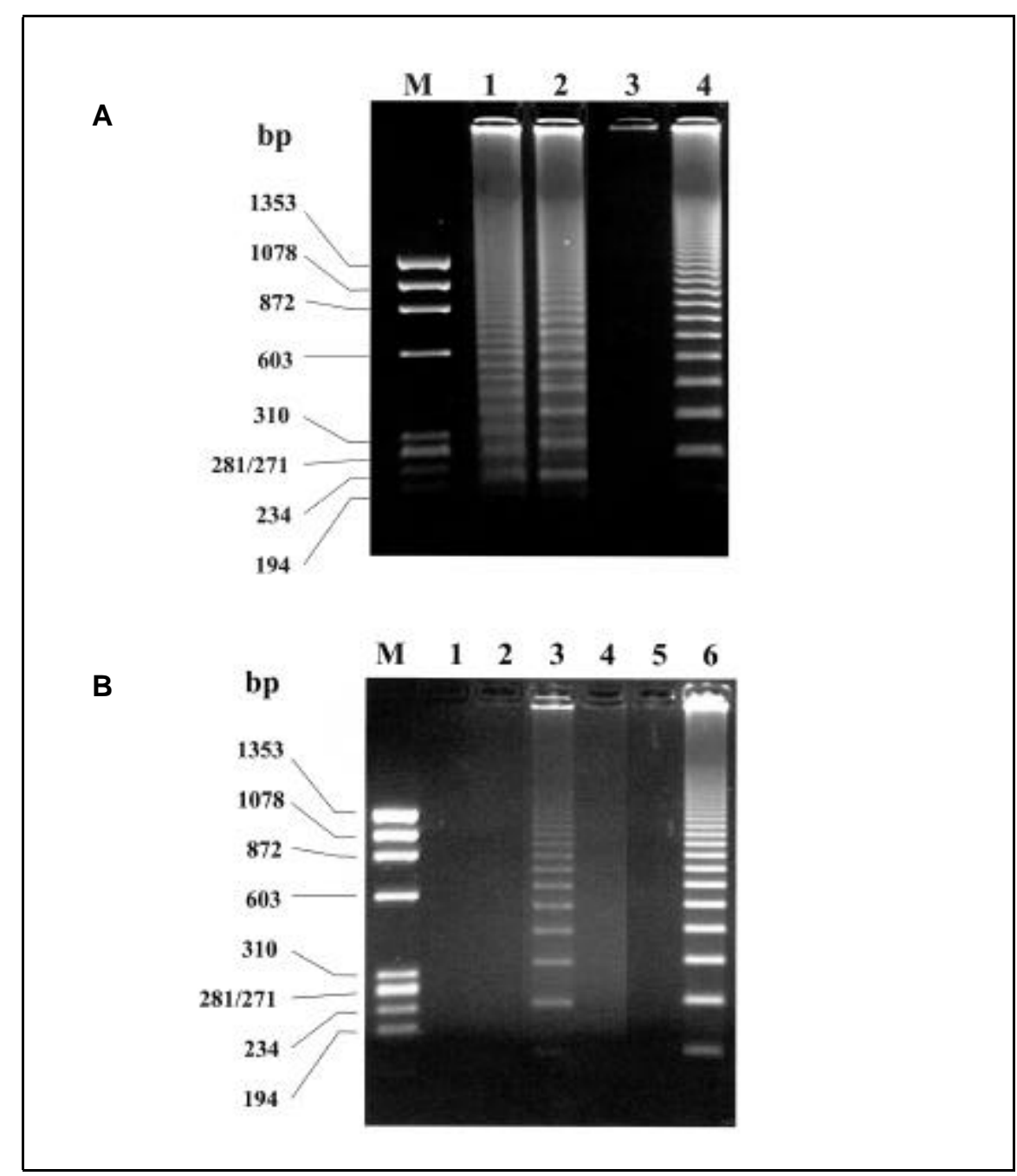

Figure 7. CRCA from affinity-purified circular DNA templates. The amplification products were electrophoresed in 2\% agarose. The DNA species used are listed in the Materials and Methods section. (A) The effect on background of circular template purification using superparamagnetic beads was determined. Lane M, molecular weight markers; lane 1, no target or ligase added annealing and ligation reaction, no affinity purification; lane 2 , standard annealing and ligation reaction, no affinity purification; lane 3 , as for lane 1 , with affinity purification step; lane 4 , as for lane 2 , with affinity purification step. (B) Background-free gene detection in a human genomic sample using circular template purification in a streptavidin-coated microplate was demonstrated. Lane M, molecular weight markers; lane 1, $200 \mathrm{ng}$ human genomic DNA, no ligase added to annealing and ligation reaction; lane 2, $100 \mathrm{ng}$ E. coli DH5 $\alpha$ genomic DNA used as target; lane 3, 200 ng human genomic DNA used as target; lane 4, 20 ng human genomic DNA used as target; lane 5, no target added; and lane 6,1 pmol synthetic target. 
merizes and exponentially amplifies both circular and linear DNA with no need for temperature cycling or additional enzymes. The potential applications of both reactions in DNA-based diagnostics are worth investigating fully. While there is no doubt that CRCA is more sensitive and predictable than LIMA, the necessity of using a padlock or similar device before amplification adds inherent complexity to CRCAbased embodiments. This complexity is accentuated by the background problems caused by LIMA and the measures needed to avoid this. In contrast, LIMA is extremely simple and straightforward to carry out - all that is required are the primers and the polymerase.

\section{ACKNOWLEDGMENTS}

This work was supported by the Cooperative Research Centre for Diagnostic Technologies. The authors thank Nancy Kleckner (Harvard University) for providing strains and Lynn Sly (School of Life Sciences, Queensland University of Technology) for the construction of the mini-transposon poolate.

\section{REFERENCES}

1.Baner, J., M. Nilsson, M. Mendel-Hartvig, and U. Landegren. 1998. Signal amplification of padlock probes by rolling-circle replication. Nucleic Acids Res. 15:5073-5078.

2.Dana, S.L., P.A. Hoener, D.A. Wheeler, C.B. Lawrence, and D.P. McDonnell. 1994. Novel estrogen response elements identified by genetic selection in yeast are differently responsive to estrogens and anitestrogens in mammalian cells. Mol. Endocrinol. 8:1193-1207.

3.Daubendiek, S.L. and E.T. Kool. 1997. Generation of catalytic RNAs by rolling-circle transcription of synthetic DNA nanocircles. Nat. Biotechnol. 15:273-277.

4.Fire, A. and S-Q. Xu. 1995. Rolling replication of short DNA circles. Proc. Natl. Acad. Sci. USA 92:4641-4645.

5.Hatch, A., T. Sano, J. Misasi, and C.L. Smith. 1999. Rolling-circle amplification of DNA im mobilised on solid surfaces and its application to multiplex mutation detection. Genet. Anal. 15:35-40.

6.Hemat, F. and K. McEntee. 1994. A rapid and efficient PCR-based method for synthesizing high-molecular-weight multimers of oligonucleotides. Biochem. Biophys. Res. Commun. 205:475-481.

7.Kadonaga, J.T. and R. Tjian. 1986. Affinity purification of sequence-specific DNA binding proteins. Proc. Natl. Acad. Sci. USA 83:58895893.
8.Kleckner, N., J. Bender, and S. Gottesman. 1991. Uses of transposons with emphasis on Tn10. Methods Enzymol. 204:139-180.

9.Krupp, G. 1989. Unusual promoter-independent transcription reactions with bacteriophage RNA polymerases. Nucleic Acid Res. 15:30233036.

10.Lee, J.H., P.M. Skowron, S.M. Rutkowska, S.S. Hong, and S.C. Kim. 1996. Sequential amplification of cloned DNA as tandem multimers using class-IIS restriction enzymes. Genet. Anal. 13:139-145.

11.Liu, D., S.L. Daubendick, M.A. Zillman, K. Ryan, and E.T. Kool. 1996. Rolling-circle DNA synthesis: small circular oligonucleotides as efficient templates for DNA polymerases. J. Am. Chem. Soc. 118:1587-1594.

12.Lizardi, P.M., X. Huang, Z. Zhu, P. BrayWard, D.C. Thomas, and D.C. Ward. 1998. Mutation detection and single-molecule counting using isothermal rolling-circle replication. Nat. Genet. 19:225-232.

13.Nillson, M., H. Malmgren, M. Samiotaki, M. Kwiatkowski, B.P. Chowdary, and U. Landegren. 1994. Padlock probes: circularising oligonucleotides for localised DNA detection. Science 265:2085-2088.

14. Rosenfeld, P.J. and T.J. Kelly. 1986. Purification of nuclear factor I by DNA recognition site affinity chromatography. J. Biol. Chem. 261:1398-1408.

15.Sambrook, J., E.F. Frisch, and T. Maniatis. 1989. Molecular Cloning: A Laboratory Manual. CSH Laboratory Press, Cold Spring Harbor, NY.

16.Schafer, A.J. and R.E. Fournier. 1992. Multiple elements regulate phosphoenolpyruvate carboxykinase gene expression in hepatoma hybrid cells. Somat. Cell. Mol. Gen. 18:571581.

17.Silhavy, T.J., M.L. Berman, and L.W. Enquist. 1984. Experiments with Gene Fusions. CSH Laboratory Press, Cold Spring Harbor, NY.

18.Thomas, D.C., G.A. Nardone, and S.K. Randall. 1999. Amplification of padlock probes for DNA diagnostics by cascade rolling-circle am plification or the polymerase chain reaction. Arch. Pathol. Lab. Med. 123:1170-1176.

19.Zhang, D.Y., M. Brandwein, T.C. Hsuih, and H. Li. 1998. Amplification of target-specific, ligation-dependent circular probe. Gene $211: 277-285$

20.Zhang, W.W., J. Farres, and H. Busch. 1991. DNA footprint enhancement using tandem binding sites. BioTechniques 1 1:728-733.

Received 26 May 2000; accepted 11 September 2000.

\section{Address correspondence to}

Dr. Phil M. Giffard

CRC for Diagnostic Technologies

Queensland University of Technology

Gardens Point Campus

GPO Box 2434

Queensland 4001, Australia

e-mail:p.giffard@qut.edu.au 